\title{
Dynamical Theory of Rubber Elasticity
}

\author{
S. F. EDWARDS \\ Cavendish Laboratory, Cambridge CB3 OHE, U.K.
}

(Received August 20, 1984)

\begin{abstract}
As an alternative to the derivation of the elastic free energy of a network by statistical mechanics, a derivation is given directly from equations of motion. It is shown that this method incorporates calculations of the visco-elastic behaviour of rubbers and shows that permanent cross links and entanglements enter the formalism in quite different ways, though both in the end contribute to the modulus.
\end{abstract}

KEY WORDS Polymer Dynamics / High Frequency Modulus / Rubber

Elasticity /

\section{THE TWO APPROACHES TO STATISTICAL PHYSICS}

There are two routes to the analysis of problems of statistical physics. The first, brought to completion by Gibbs, discusses equilibrium thermodynamic functions; the typical formula is that the free energy $A$ is given by an integration using the Hamiltonian $H$

$$
\exp [-A / \kappa T]=\int \exp [-H / \kappa T] \mathrm{d}(\text { phase space }) \text {. }
$$

This method allows one to study many problems with great accuracy, e.g., the virial series for an imperfect gas, and offers a base for the study of critical phenomena. The other method pioneered by Boltzmann is to derive equations of motion for statistical averages and from these produce transport equations, in which can be found not only the thermodynamic functions, but also transport coefficients. In principle therefore the second method includes all the results of the first, and gives physical quantities not available from the Gibbsian method. In practice one finds the second method much more difficult than the first, so for example, the virial series for the imperfect gas has yet to be developed past the first term via the Boltzmann equation.

When constraints, like permanent cross links in a rubber network, are added, the Gibbs formalism becomes much more complicated, and an extraor- dinary richness of behaviour is being discovered in problems like spin glasses. For rubbers however it seems that one can make good progress, although little attention has been paid in the literature to the fact that a complete formalism is now available. The difficulty with the Gibbs approach is that if a set of cross links is introduced into a system, and these are permanent, then the free energy must be a function of the specification of these cross links. Thus if the $i$ th polymer is labelled $\boldsymbol{R}_{i}\left(s_{i}\right), s_{i}$ labelling the $s_{i}$ th link (to be treated as a continuous variable from now on), and if chain $i$ meets $j$ at $s_{i}^{j}$, also $j$ meets $i$ at $s_{j}^{i}$, then the free energy $A$ is $A([\mathrm{~s}])$, [s] denoting the matrix $s_{i}^{j}$. For a macroscopic body the experimental free energy is

$$
\bar{A}=\int A([\mathrm{~s}]) P([\mathrm{~s}]) \mathrm{d}[\mathrm{s}]
$$

where $P([\mathrm{~s}])$ is the probability distribution of the cross links, a function laid down when the network is created. Hence if we assume the links are suddenly imposed, the simplest assumption to make,

$$
\begin{aligned}
\bar{A}= & \int P([\mathrm{~s}])\left(\operatorname { l o g } \int \operatorname { e x p } [ - H / \kappa T ] \prod _ { [ \mathrm { s } ] } \delta \left(\boldsymbol{R}_{i}\left(s_{i}^{j}\right)\right.\right. \\
& \left.\left.-\boldsymbol{R}_{j}\left(s_{j}^{i}\right)\right) \mathrm{d}(\text { polymer configurations })\right) \mathrm{d}[\mathrm{s}]
\end{aligned}
$$

A trick to put this in an elegant way is to use replication, ${ }^{1}$ i.e., to regard the logarithm in this way:

$$
X^{n}=1+n \log X+\cdots
$$




\section{S. F. EdWARDS}

so imagine our system repeated $n$ times, with polymer coordinates

$$
\begin{aligned}
& R^{(1) \cdots} R^{(n)} \\
& \qquad \begin{array}{l}
\log \left(\int \exp [-H / \kappa T] \prod \delta\right)=\text { coeff. of } n \text { in } \\
\quad \int \cdots \prod_{\alpha=1}^{n} \mathrm{~d} R^{(\alpha)} \exp \left[-\sum_{1}^{n} H^{(\alpha)} / \kappa T\right] \prod_{\alpha=1}^{n} \prod_{[\mathrm{s}]} \\
\quad \times \delta\left(R_{i}^{(\alpha)}-R_{j}^{(\alpha)}\right)
\end{array}
\end{aligned}
$$

The simplest form for $P$ is to say the links were imposed on equilibrium, so $P$ also has the form

$$
\begin{aligned}
P([\mathrm{~s}])= & \int \exp \left[\left(A^{(0)}-H^{(0)}\right) / \kappa T\right] \prod_{[\mathrm{s}]} \delta\left(R_{i}^{(0)}\left(s_{i}^{j}\right)\right. \\
& \left.-R_{j}^{(0)}\left(s_{j}^{i}\right)\right)\left(\mathrm{d} \boldsymbol{R}^{(0)}\right)
\end{aligned}
$$

where $\left(\mathrm{d} \boldsymbol{R}^{(0)}\right)$ means integration over all configurations of $\boldsymbol{R}^{(0)}(s)$.

At this point one can use the grand canonical ensemble with a potential conjugate to the number of cross links so that finally one can introduce

$$
\begin{aligned}
& \exp [-A(n) / \kappa T]=\int \frac{\mathrm{d} \mu}{N_{c} !} \exp \left[N_{c} \log \mu-\sum_{0}^{n} H^{(\alpha)} / \kappa T\right. \\
&\left.+\mu \int_{0}^{i} \int_{0}^{i} \mathrm{~d} s_{1} \mathrm{~d} s_{2} \prod_{\alpha=0}^{n} \delta\left(R_{\left(s_{1}\right)}^{(\alpha)}-R_{\left(s_{2}\right)}^{(\alpha)}\right)\right] \\
& A(n)=A(0)+n \bar{A}+O\left(n^{2}\right) .
\end{aligned}
$$

When the system is distorted, the integral is taken over

$$
\left\{\begin{array}{l}
\text { space }(0) \text { original system } \\
\text { spaces }(1) \cdots(n) \text { strained system } .
\end{array}\right.
$$

This gives a complete formalism and can be the basis of approximations.

So far there are no entanglements. Introducing them is a problem since there is no complete mathematical theory specifying the topological relationship of curves. But suppose for the moment that we did have this specification, or argue that the Gaussian integral invariant ${ }^{2} I_{i j}$ is good enough. Then in the original system $I_{i j}$ will take on values $J_{i j}$ say. These same values will be preserved in the strained system, so one has to modify eq 6 by adding a factor

$$
\delta\left(I_{i j}^{(0)}-J_{i j}\right) \delta\left(J_{i j}-I_{i j}^{(\alpha)}\right)
$$

$$
\prod_{i, j} \prod_{\alpha} \delta\left(I_{i j}^{(\alpha)}-I_{i j}^{(0)}\right)
$$

where the product $i, j$ is over all pairs of chains.

With this proviso about the completeness of the entanglement description, the Gibbs formalism is complete.

If we now turn to the Boltzmann approach, the particular version suitable for polymer problems is the Langevin-Smoluckowski approach rather than the original Boltzmann equation. The constraints of the network can be incorporated into dissipative equations by using the Rayleigh friction formalism, and the entanglements can be built in by noting that the coupled equations for network and solvent conserve topological invariants. If there is no solvent, the matrix of chains itself will serve this purpose.

We will approach the problem by a hierarchy of approximations,

(i) The dynamics of a simple cross linked network.

(ii) The equations of elasticity from this network.

(iii) The dynamics of a network embedded in a solvent.

(iv) The visco-elastic equations of the rubber.

\section{THE DYNAMICS OF A SIMPLE NETWORK}

The simplest dynamics of a single chain lies in the Rouse equation, which in the Langevin form, is

$$
v \dot{\boldsymbol{R}}(s, t)-\frac{3 \kappa T}{b} \frac{\partial^{2} \boldsymbol{R}(s, t)}{\partial s^{2}}=f(s, t)
$$

which can be derived from the Rayleigh function ${ }^{3}$

$$
\left.\frac{1}{2} v \int \boldsymbol{U}^{2} \mathrm{~d} s \mathrm{~d} t+\frac{3}{2} \frac{\kappa T}{b} \int \boldsymbol{R}^{\prime 2} \mathrm{~d} s \mathrm{~d} t+\int \Phi(\boldsymbol{R} / s, t)\right) \mathrm{d} s \mathrm{~d} t
$$

where $\boldsymbol{U}$ is $\dot{\boldsymbol{R}}$ but is independently varied as is done in the use of friction functions.

To add the effects of cross links, one uses Langrange multipliers $p_{i j}$ and adds

$$
\sum_{i j} \boldsymbol{p}_{i j}\left\{\boldsymbol{R}_{i}\left(s_{i}^{j}\right)-\boldsymbol{R}_{j}\left(s_{j}^{i}\right)\right\}
$$

which modifies the equations to

or equivalently 


$$
v \dot{\boldsymbol{R}}_{i}-\frac{3 \kappa T}{b} \boldsymbol{R}_{i}^{\prime \prime}+\sum_{j} p_{i j} \delta\left(s-s_{i}^{j}\right)=\boldsymbol{f}_{i} .
$$

One can expect the cross links to pin the chain down to some mean position $\overline{\boldsymbol{R}}$ which can also be regarded as the mean position of other chains which are in the neighbourhood of $\boldsymbol{R}_{i}$. Thus we expect an average behaivour of eq 12 to look like

$$
v \dot{\boldsymbol{R}}_{i}-\frac{3 \kappa T}{b}\left\{\boldsymbol{R}_{i}^{\prime \prime}-q_{0}^{2}\left(\boldsymbol{R}_{i}-\overline{\boldsymbol{R}}\right)\right\}=\boldsymbol{f}_{i}
$$

or in Fourier (i.e., Rouse variable) transform

$$
v \dot{\boldsymbol{R}}_{i q}+\frac{3 \kappa T}{b}\left\{q^{2} \boldsymbol{R}_{i q}+q_{0}^{2}\left(\boldsymbol{R}_{i q}-\overline{\boldsymbol{R}}_{i q}\right)\right\}=\boldsymbol{f}_{i q} .
$$

In fact for a well cross linked network we can ignore end effects and imagine the whole system as one long chain, so dropping the $i$ on $\boldsymbol{R}$, the cross links now being at points $s^{i}, s^{j}$. We are expecting therefore that

$$
\sum p_{i j}\left\{\delta\left(s-s^{i}\right)-\delta\left(s-s^{j}\right)\right\}
$$

becomes

$$
q_{0}^{2}(\boldsymbol{R}-\overline{\boldsymbol{R}})
$$

So let us write

$$
\begin{aligned}
& v \dot{R}_{q}+\frac{3 \kappa T}{b}\left\{q^{2} \boldsymbol{R}_{q}+q_{0}^{2}\left(\boldsymbol{R}_{q}-\bar{R}_{q}\right)\right\} \\
& =\left(\frac{3 \kappa T}{b} q_{0}^{2}\left(\boldsymbol{R}_{q}-\overline{\boldsymbol{R}}_{q}\right)-\sum p_{i j}\left\{\delta\left(s-s^{i}\right)-\delta\left(s-s^{j}\right)\right\}\right),
\end{aligned}
$$

and isolate each link, treating all the others as if the $\sum p \delta$ term were already $q_{0}^{2}(\boldsymbol{R}-\overline{\boldsymbol{R}})$. Then one has, if

$$
\left.\begin{array}{c}
\left(v \frac{\partial}{\partial t}+\frac{3 \kappa T}{b}\left(q^{2}+q_{0}^{2}\right)\right) G=\delta\left(t-t^{\prime}\right) \\
\left(i v \omega+\frac{3 \kappa T}{b}\left(q^{2}+q_{0}^{2}\right)\right) G=1 \\
\left(v \frac{\partial}{\partial t}+\frac{3 \kappa T}{b}\left(q_{0}^{2}-\frac{\partial^{2}}{\partial s^{2}}\right)\right) G=\delta\left(s-s^{\prime}\right) \delta\left(t-t^{\prime}\right)
\end{array}\right\} \text { (18) }
$$

where $\boldsymbol{R}_{0}$ is the position taken by the polymer if the $i, j$ link is absent. Since $\boldsymbol{R}\left(s^{i}\right)-\boldsymbol{R}\left(s^{j}\right)=0$

$$
\begin{aligned}
0 & =\boldsymbol{R}_{0}\left(s^{i}\right)-\boldsymbol{R}_{0}\left(s^{j}\right) \\
& +\int G\left(s^{i}, s^{\prime \prime} ; \omega\right) \frac{3 \kappa T}{N_{c} b} q_{0}^{2}\left\{\boldsymbol{R}\left(s^{\prime \prime}, \omega\right)-\overline{\boldsymbol{R}}\left(s^{\prime \prime}, \omega\right)\right\} \mathrm{d} s^{\prime \prime} \\
& -\sum\left[G\left(s^{i}, s^{i} ; \omega\right)+G\left(s^{j}, s^{j} ; \omega\right)-G\left(s^{i}, s^{j} ; \omega\right)\right. \\
& \left.-G\left(s^{j}, s^{i} ; \omega\right)\right] p_{i j}(\omega)
\end{aligned}
$$

Now sum over $j$.

Of these terms it is easy to show that the dominant term $g\left(s^{i}, s^{i} ; \omega\right), g\left(s^{j}, s^{j} ; \omega\right)$ i.e.,

$2 \cdot \frac{1}{2 \pi} \cdot \int \frac{\mathrm{d} q}{i v \omega+\frac{3 \kappa T}{b}\left(q^{2}+q_{0}^{2}\right)}=\frac{b}{3 \kappa T}\left(q_{0}^{2}+\frac{i v \omega b}{3 \kappa T}\right)^{1 / 2}$

One can now argue that the average of $\boldsymbol{R}_{0}\left(s^{j}\right)$ is just $\overline{\boldsymbol{R}}$ and hence identify $p$ with

$$
\frac{b}{3 \kappa T}\left(q_{0}^{2}+\frac{i v \omega b}{3 \kappa T}\right)
$$

Hence one has, if the density of cross links per unit length of polymer is $n_{\mathrm{c}}$

$$
q_{0}^{2}=n_{c} \sqrt{q_{0}^{2}+\frac{i v \omega b}{3 \kappa T}}
$$

or

$$
\begin{aligned}
q_{0}^{2} & =n_{c} q_{0}+\frac{i v \omega b}{6 \kappa T q_{0}} \\
& =n_{c}+\frac{i v \omega b}{6 \kappa T n_{c}} .
\end{aligned}
$$

Returning now to the original equation one finds

$$
2 i v \omega \boldsymbol{R}-\frac{3 \kappa T}{b}\left\{q^{2} \boldsymbol{R}+q_{0}^{2}(\boldsymbol{R}-\overline{\boldsymbol{R}})\right\}=\boldsymbol{f}
$$

or putting

$$
\begin{gathered}
\boldsymbol{R}-\overline{\boldsymbol{R}}=\boldsymbol{r} \\
2 i v \omega \boldsymbol{r}+\frac{3 \kappa T}{b}\left(q^{2}+q_{0}^{2}\right) \boldsymbol{r}=\boldsymbol{f} .
\end{gathered}
$$

Thus we have shown that the polymer is tied by the cross links to sit in an effective tube characterized by $q_{0}$ the arc density of links and, within the tube, the frictional effects are doubled. The radius of the tube varies with $q_{0}^{-1 / 2}$, i.e., $n_{\mathrm{c}}^{-1 / 2}$. 


\section{S. F. EDWARDS}

\section{THE EQUATIONS OF ELASTICITY FOR THE NETWORK ${ }^{4,5}$}

To obtain the equations for an elastic deformation $\boldsymbol{u}(\boldsymbol{r}, t)$ we have to write the position of the polymer as

$$
R=R_{0}+u(R)
$$

where $\boldsymbol{R}_{0}$ now denotes position before deformation, and modify our equations of motion to ensure that friction takes place proportional not to $\dot{\boldsymbol{R}}$ but $\dot{\boldsymbol{R}}-\dot{\boldsymbol{u}}$ and similarly the tube constraint acts now on $\boldsymbol{R}-\boldsymbol{u}$. We also must include inertia in the formalism, and eventually have (putting $\varepsilon=3 \kappa T / b$ )

$$
m \ddot{\boldsymbol{R}}+v(\dot{\boldsymbol{R}}-\dot{\boldsymbol{u}})-\varepsilon\left\{\boldsymbol{R}^{\prime \prime}-q_{0}^{2}\left(\boldsymbol{R}-\boldsymbol{u}-\boldsymbol{R}_{0}\right)\right\}=\boldsymbol{f}
$$

which presents the difficulty that $\boldsymbol{u}$ is $\boldsymbol{u}(\boldsymbol{R})$ not $\boldsymbol{u}\left(\boldsymbol{R}_{0}\right)$. If we assume that we can just leave $\boldsymbol{R}$ in $\boldsymbol{u}$ without allowing for its motion (which will prove a similar approximation to that normally used) we can also notice that the condition that the system without stress is in equilibrium means that when $\boldsymbol{u}=0, \boldsymbol{R}$ should be $\boldsymbol{R}_{0}$ apart from fluctuations, which is correct. Solve the equation by now writing in Fourier transform

$$
\begin{aligned}
\boldsymbol{R}-\boldsymbol{R}_{0}= & \sum_{\boldsymbol{j}} \frac{\left(i v \omega+\varepsilon q_{0}^{2}\right) \boldsymbol{u}_{\boldsymbol{j}}(t) \exp \left[i \boldsymbol{R}_{\boldsymbol{j}}(s, t)+i \omega t+i q s\right]}{i v \omega+\varepsilon\left(q^{2}+q_{0}^{2}\right)+m \omega^{2}} \\
& +\frac{\boldsymbol{f}}{i v \omega+m \omega^{2}+\varepsilon\left(q^{2}+q_{0}^{2}\right)}
\end{aligned}
$$

But

$$
\boldsymbol{u}_{k}=\int \mathrm{d}^{3} \boldsymbol{k} \mathrm{e}^{i \boldsymbol{k} \cdot \boldsymbol{R}(s t)}\left\{\boldsymbol{R}(s t)-\boldsymbol{R}_{0}(s t)\right\} \mathrm{d} s
$$

Therefore we define the macroscopic force $\boldsymbol{F}_{\boldsymbol{k}}$ by an average over $f$ and average

$$
\left\langle\mathrm{e}^{i \boldsymbol{k} \cdot \boldsymbol{R}(s, t)+i \boldsymbol{j} \cdot \boldsymbol{R}\left(s^{\prime}, t^{\prime}\right)}\right\rangle
$$

by writing

$$
\begin{aligned}
\boldsymbol{R}(s, t)-\boldsymbol{R}\left(s^{\prime}, t^{\prime}\right) & \cong \boldsymbol{R}(s, t)-\boldsymbol{R}\left(s^{\prime}, t\right) \\
& =\boldsymbol{E} \cdot\left(\boldsymbol{R}_{0}(s)-\boldsymbol{R}_{0}\left(s^{\prime}\right)\right)
\end{aligned}
$$

where $\boldsymbol{E}$ is the strain tensor, so that

$$
\left\langle\mathrm{e}^{i \boldsymbol{k} \cdot\left\{\boldsymbol{R}(s, t)-\boldsymbol{R}\left(s^{\prime}, t^{\prime}\right)\right\}}\right\rangle=\exp \left[-\frac{b}{6}(\boldsymbol{k} \cdot \boldsymbol{E} \cdot \boldsymbol{E} \cdot \boldsymbol{k})\left|s-s^{\prime}\right|\right]
$$

$$
\begin{aligned}
& \left\{m \omega^{2}+i v \omega+\varepsilon\left(q^{2}+q_{0}^{2}\right)\right\}^{-1} \\
& =\frac{1}{2} \varepsilon^{-1 / 2}\left(i v \omega+\varepsilon q_{0}^{2}+m \omega^{2}\right)^{-1 / 2} \\
& \quad \times \exp \left[-\left(q_{0}^{2}+\frac{m \omega^{2}}{\varepsilon}+\frac{i v \omega}{\varepsilon}\right)^{1 / 2}\left|s-s^{\prime}\right|\right]
\end{aligned}
$$

which allows the integrals to be performed and leaves

$$
\begin{aligned}
\boldsymbol{u}_{\boldsymbol{k}}= & m \boldsymbol{F}_{\boldsymbol{k}}\left(\varepsilon q_{0}^{2}+i v \omega+m \omega^{2}\right)^{-1} \\
+ & \frac{1}{2}\left(q_{0}^{2}+\frac{i v \omega}{\varepsilon}\right)\left(q_{0}^{2}+\frac{i \nu \omega}{\varepsilon}+\frac{m \omega^{2}}{\varepsilon}\right)^{-1 / 2} \\
& \times\left(q_{0}^{2}+\frac{i v \omega}{\varepsilon}+\frac{m \omega^{2}}{\varepsilon}+\frac{\boldsymbol{k} \boldsymbol{E} \boldsymbol{E} \boldsymbol{k}}{6 b}\right)^{-1 / 2} \boldsymbol{u}_{\boldsymbol{k}}
\end{aligned}
$$

$$
\begin{gathered}
\left(\varepsilon q_{0}^{2}+i v \omega+m \omega^{2}-\varepsilon q_{0}^{2}-i v \omega-\frac{\varepsilon b}{6}(\boldsymbol{k E E k})\right. \\
\left.\times\left(q_{0}^{2}+\frac{i v \omega}{\varepsilon}+\frac{m \omega^{2}}{\varepsilon}\right)^{1 / 2}\right) u_{k \omega}=F_{k \omega} \\
\left\{\omega^{2}-(\boldsymbol{k E E E k}) \Gamma(\omega)\right\} u_{k \omega}=F_{k \omega} .
\end{gathered}
$$

We have therefore derived the equations for elasticity which are brought into conventional form if $\boldsymbol{E}$ is replaced by the unit matrix, and only terms of order $k^{2} \omega$ retained in addition to the usual wave terms, i.e.,

$$
\frac{\partial^{2} \boldsymbol{u}}{\partial t^{2}}-c^{2} \nabla^{2} \boldsymbol{u}-\frac{i v b}{6 \varepsilon q_{0}} \nabla^{2} \frac{\partial \boldsymbol{u}}{\partial t}=\boldsymbol{F}
$$

where

$$
c^{2}=\frac{b q_{0}}{6 m} \varepsilon \quad \text { and } \quad \frac{v b}{\varepsilon q_{0}}
$$

represents the viscosity of the system. This analysis has not taken into account the compressibility of the network, but it is straightforward to add this in, since the osmotic free energy is $\mathscr{A}\left(\boldsymbol{E}^{2}\right)$ and this adds directly a term which at the lowest order is just $\boldsymbol{V} p$.

Although we have given a very brief outline here of the derivation it should be clear that given microscopic equations of motion, one can readily transfer to macroscopic equations, simply because polymer interactions are long range and of a basically simple structure.

So far we have only considered networks, but must now progress to entanglements.

But in $s$ space 


\section{THE PROBLEM OF ENTANGLEMENTS}

It is important to realise how entanglements differ from cross links. We have seen that a set of cross links can be represented by a Rouse equation.

$$
\nu \dot{\boldsymbol{R}}+\varepsilon q^{2} \boldsymbol{R}+\varepsilon q_{0}^{2}(\boldsymbol{R}-\overline{\boldsymbol{R}})=\boldsymbol{f}
$$

and if $f$ is a Langevin random force this is equivalent to

$$
\begin{aligned}
\left(\frac{\partial}{\partial t}-\sum_{q} \frac{\partial}{\partial \boldsymbol{R}_{q}} \frac{\kappa T}{v}\left(\frac{\partial}{\partial \boldsymbol{R}_{-q}}+\frac{3}{b} q^{2} \boldsymbol{R}_{q}\right.\right. \\
\left.\left.+\frac{3}{b} q_{0}^{2}\left(\boldsymbol{R}_{q}-\overline{\boldsymbol{R}}_{q}\right)\right)\right) P=0 .
\end{aligned}
$$

The solution of this, i.e., the equilibrium of eq 39 is given by

$$
\exp \left(-\frac{\varepsilon}{2} \sum q^{2}\left|\boldsymbol{R}_{q}\right|^{2}-\frac{\varepsilon}{2} q_{0}^{2} \sum\left|\left(\boldsymbol{R}_{q}-\overline{\boldsymbol{R}}_{q}\right)\right|^{2}\right)
$$

and this represents a Gaussian random walk constructed to be close to some mean position. It is clearly not the same as a free polymer which will only have the usual

$$
\exp \left(-\frac{\varepsilon}{2 \kappa T} \sum_{q} q^{2}\left|\boldsymbol{R}_{q}\right|^{2}\right)
$$

But now consider a polymer which, for simplicity, is added to solution containing an existing network, a simpler still to a solution in which all polymers but itself are frozen. All states are now accessible, but clearly the motion is inhibited and in melt conditions will be reptation. The free polymer satisfies the equation given by Rouse

$$
\left(\frac{\partial}{\partial t}-\sum_{q} \frac{\partial}{\partial \boldsymbol{R}_{q}} \frac{\kappa T}{v}\left(\frac{\partial}{\partial \boldsymbol{R}_{-q}}+\frac{3 q^{2}}{b} \boldsymbol{R}_{q}\right)\right) P=0 .
$$

To handle entanglements one can try to put a 'tube' potential as in eq 40 but it is not obvious how to do it, since the equilibrium solution is indeed eq 42 . A way around this problem is to consider the more general equations including hydrodyamic interactions. Let $G_{i j}\left(\boldsymbol{R}_{\alpha}, \boldsymbol{R}_{\beta}\right)$ be the Oseen tensor ${ }^{6}$

$$
\int \frac{\mathrm{d}^{3} k}{k^{2}}\left(\delta_{i j}-\frac{k_{i} k_{j}}{k^{2}}\right) \exp \left[i \boldsymbol{k} \cdot\left(\boldsymbol{R}_{\alpha}-\boldsymbol{R}_{\beta}\right)\right]
$$

Then for an assembly of polymers ${ }^{6}$

$$
\begin{gathered}
\left(\frac{\partial}{\partial t}-\frac{\kappa T}{\eta} \sum_{\alpha, \beta} \int \mathrm{d} s_{\alpha} \int \mathrm{d} s_{\beta} \frac{\partial}{\partial R_{\alpha i}\left(s_{\alpha}\right)} G_{i j}\left(\boldsymbol{R}_{\alpha}, \boldsymbol{R}_{\beta}\right)\right. \\
\left.\times\left(\frac{\partial}{\partial R_{\beta j}\left(s_{\beta}\right)}+\frac{3}{b} \frac{\partial^{2} R_{\beta j}}{\partial s_{\beta}^{2}}\right)\right) P=0
\end{gathered}
$$

where $\eta$ is the viscosity of the surrounding fluid.

A remarkable property of this equation is that no chain passes through any other in the course of its diffusion. In spite of the apparent divergence of the Oseen tensor, when carefully evaluated the effective diffusion constant of one chain say $\boldsymbol{R}_{1}$, when the others are fixed, vanishes as $\boldsymbol{R}_{1} \rightarrow \boldsymbol{R}_{i}$. The vanishing of the diffusion constant is sufficient to ensure that $\boldsymbol{R}_{1}$ never passes any $\boldsymbol{R}_{i}$. Imagine the mean locus of $\boldsymbol{R}_{1}$ with all the others fixed is $\boldsymbol{R}$ (i.e., disregard reptation for the moment). Then the average diffusion constant will vanish like $\left(\log |\boldsymbol{R} \times \boldsymbol{t}| q_{0}\right)^{-1}$ where $t$ is the tangent to the mean locus and $q_{0}^{-1 / 2}$ the radius of the tube (the mean locus is called the primitive path, and it is smoother than the random walk of the polymer, so that a tangent to it exists which is not the case for a Gaussian walk). Thus we can now study the Green function of eq 45 , with all but one polymer fixed, and introduce the primitive path this way: Let the Green function relate the probability of finding $\boldsymbol{R}$ at $t$ and $\tilde{\boldsymbol{R}}$ at $\tilde{t}$. Then

$$
\begin{gathered}
\left(\frac{\partial}{\partial t}-\int \mathrm{d} s \frac{\partial}{\partial R} D(\boldsymbol{R}, \hat{\boldsymbol{R}})\left(\frac{\partial}{\partial \boldsymbol{R}}+\frac{3}{b} \frac{\partial^{2} \boldsymbol{R}}{\partial s^{2}}\right)\right) \boldsymbol{G}(\boldsymbol{R}, \tilde{\boldsymbol{R}}) \\
=\delta(t-\tilde{t}) \prod_{s} \delta(\boldsymbol{R}(s)-\tilde{\boldsymbol{R}}(s))
\end{gathered}
$$

where, if

$$
\tilde{\boldsymbol{R}}(s)=\sum_{q=0}^{\infty} \tilde{\boldsymbol{R}}_{q} \mathrm{e}^{i q s}
$$

then its primitive path is

$$
\hat{\boldsymbol{R}}(s)=\sum_{q=0}^{q_{0}} \tilde{\boldsymbol{R}}_{q} \mathrm{e}^{i q s}
$$

$D$ is now a constant along $\boldsymbol{R}$, but goes to zero at a perpendicular tube width.

The form of the equation is rather like that encountered in plasma physics where one finds $D_{\|}$ and $D_{\perp}$ for the diffusion along and perpendicular to magnetic fields.

Thus we now have a closed exact equation for entangled diffusion eq 45 and a model for practical calculations. For high concentrations $D_{\perp}$ can be ignored and the resulting visco-elastic behaviour is 
much simplified and is given by Doi and Edwards. ${ }^{7}$

Now we can approach the central problem. If cross links and entanglements are present what do we do? The effect of the diffusion coefficient structure is to break up the equilibrium distribution into its topological classes, inaccessible to one another if $D$ vanishes at their boundary, i.e., as chains touch. This must happen also when cross linkage entraps entanglements, so the complete diffusion equation is

$$
\begin{gathered}
\left(\frac{\partial}{\partial t}-\frac{\kappa T}{\eta} \iint \frac{\partial}{\partial \boldsymbol{R}_{\alpha}} \boldsymbol{G}\left(\boldsymbol{R}_{\alpha} \boldsymbol{R}_{\beta}\right)\left(\frac{\partial}{\partial \boldsymbol{R}_{\beta}}+\frac{3}{b} \frac{\partial^{2} \boldsymbol{R}_{\beta}}{\partial s_{\beta}{ }^{2}}\right.\right. \\
\left.\left.+\frac{3 q_{0}^{2}}{b}\left(\boldsymbol{R}_{\beta}-\tilde{\boldsymbol{R}}_{\beta}\right)\right)\right) \boldsymbol{G}=\Pi \delta
\end{gathered}
$$

It is now instructive to go back to the Langevin equation which will be

$$
\dot{\boldsymbol{R}}_{\alpha}+\frac{3 \kappa T}{\eta b} \sum_{\beta} \int \boldsymbol{G}\left(R_{\alpha} R_{\beta}\right)\left(\boldsymbol{R}_{\beta}^{\prime \prime}-q_{0}^{2}\left(\boldsymbol{R}_{\beta}-\overline{\boldsymbol{R}}_{\beta}\right)\right)=f_{\alpha} .
$$

which contains the limits of a 'soft' environment where the many chains are adequately described by replacing the complicated Oseen tensor by a local screened interaction depending on the concentration, but if effect taking one back to the Rouse equation, to a 'hard' environment where $\boldsymbol{G}$ represents the tube of reptation. In this case of course to some extent one can argue that the case is covered by a redefinition of $q_{0}^{2}$ to cover not only the cross links, but entanglements as well, even this is not a fundamental approach. The calculation of $q_{0}^{2}$ now becomes much more complicated, but approximately is given by

$$
q_{0}=n_{\mathrm{c}}+n_{\mathrm{e}}
$$

where $n_{\mathrm{e}}$ varies like $c$, the concentration of polymer in unswollen conditions ( $c^{5 / 4}$ in scaling conditions). However it must be emphasized that both from cross links and entanglements $q_{0}$ is really frequency dependent.

\section{THE GENERAL EQUATION OF ELASTICITY}

The problem of obtaining the general equation is now clear. The microscopic equations for networks are given by eq 49 , but the entanglement comes in a subtle way, a way which loses the entanglement on simple averaging (the preaveraging approximation). Suppose nevertheless that we do this. Then one finds an effective $v$ which is proportional to $\eta q_{0}^{-1 / 2}$ and one is back to the Rouse equation with however a cross link and concentration dependent friction term.

The central problem is to go from the microscopic equation in terms of " $\boldsymbol{R}$ " to collective coordinates such as $\boldsymbol{u}$ and $\dot{\boldsymbol{u}}$ and others which do not normally appear in visco-elastic theory. This offers a major challenge to the theoretical physicist for all the keys to the solution of the problem are there

-the simple network is soluble

-the low and high concentration visco-elastic equations are soluble, and finally

- the microscopic equations are given, and are smoothly varying so that (unlike small molecule visco-elasticity) should carry over directly into macroscopic equations.

\section{REFERENCES}

1. R. T. Deam and S. F. Edwards, Phil. Trans., A280, 317 (1976).

2. S. F. Edwards, Proc. Phys. Soc., 90, 513 (1967). J. Phys. (London), Ser. A, 15 (1968). K. Iwata, J. Chem. Phys., 78, 2778 (1983). K. Iwata and T. Kimura, J. Chem. Phys., 74, 2039 (1981). F. Tanaka, Prog. Theor. Phys., 68, 148, 164 (1982).

3. S. F. Edwards and K. F. Freed, J. Chem. Phys., 61, 1189 (1974).

4. S. F. Edwards, J. Phys. (London), Ser. A, 7, 318 (1974).

5. S. F. Edwards and A. G. Miller, J. Phys., C, 9, 2001, 2011 (1976).

6. S. F. Edwards, Proc. R. Soc. London Ser. A, 385, 267 (1982).

7. M. Doi and S. F. Edwards, J. Chem. Soc., Faraday Trans. 2, 1789, 1802, and 1818 (1978). 